\title{
OPEN Reproducibility of automated habenula segmentation via deep learning in major depressive disorder and normal controls with 7 Tesla MRI
}

\author{
Sang-Heon Lim ${ }^{1,2,6}$, Jihyun Yoon ${ }^{3,6}$, Young Jae Kim², Chang-Ki Kang ${ }^{4}$, Seo-Eun Cho ${ }^{5}$, \\ Kwang Gi Kim ${ }^{1,2 \bowtie}$ \& Seung-Gul Kang ${ }^{5 凶}$
}

The habenula is one of the most important brain regions for investigating the etiology of psychiatric diseases such as major depressive disorder (MDD). However, the habenula is challenging to delineate with the naked human eye in brain imaging due to its low contrast and tiny size, and the manual segmentation results vary greatly depending on the observer. Therefore, there is a great need for automatic quantitative analytic methods of the habenula for psychiatric research purposes. Here we propose an automated segmentation and volume estimation method for the habenula in 7 Tesla magnetic resonance imaging based on a deep learning-based semantic segmentation network. The proposed method, using the data of 69 participants ( 33 patients with MDD and 36 normal controls), achieved an average precision, recall, and dice similarity coefficient of $0.869,0.865$, and 0.852 , respectively, in the automated segmentation task. Moreover, the intra-class correlation coefficient reached 0.870 in the volume estimation task. This study demonstrates that this deep learning-based method can provide accurate and quantitative analytic results of the habenula. By providing rapid and quantitative information on the habenula, we expect our proposed method will aid future psychiatric disease studies.

The habenula $(\mathrm{Hb})$ is a paired epithalamic structure adjacent to the dorsomedial thalamus and the third ventricle ${ }^{1}$ that can be divided into distinct portions via different cellular morphological features. It integrates information received from the cerebral and limbic cortex and provides forebrain control over the activity of ascending monoaminergic projections from the brainstem ${ }^{2}$. Additionally, based on previous studies of $\mathrm{Hb}$ function, the $\mathrm{Hb}$ is involved in the pathogenesis of psychiatric disorders such as major depressive disorder (MDD) ${ }^{3,4}$. Compared to normal controls (NCs), the Hb volume of patients with MDD showed atrophy in a post-mortem study $\mathrm{y}^{5}$.

According to previous post-mortem and structural imaging studies, the average volume of the human $\mathrm{Hb}$ is $15-30 \mathrm{~mm}^{35,6}$. Several studies have reported comparing the volume of the Hb between patients with a psychiatric disorder and NCs: volume comparison among patients with different stages of MDD and NCs ${ }^{7}$; among medicated and unmedicated MDD patients, bipolar disorder patients, and $\mathrm{NCs}^{8}$; and among medicated and unmedicated patients with MDD and $\mathrm{NCs}^{9}$. The majority of previous human Hb volumetric studies have used manual segmentation to determine $\mathrm{Hb}_{\mathrm{b} \text { volumes }}{ }^{7-10}$. However, these conventional manual-based approaches are time-consuming and laborious, particularly with extensive datasets, and it is challenging to accurately produce the segmented masks due to the anatomical characteristics of the Hb. Thus, manual segmentation results of the $\mathrm{Hb}$ by different observers have large deviations and it is difficult to determine which fit the gold standard. To overcome this problem, two examiners trace the individual region and the reliability of their results are evaluated

\footnotetext{
${ }^{1}$ Department of Health Sciences and Technology, Gachon Advanced Institute for Health Sciences and Technology (GAIHST), Gachon University, Seongnam-si, Republic of Korea. ${ }^{2}$ Department of Biomedical Engineering, College of Medicine, Gachon University, Seongnam-si, Republic of Korea. ${ }^{3}$ Department of Family Medicine, Yongin Severance Hospital, Yonsei University College of Medicine, Yongin, Republic of Korea. ${ }^{4}$ Department of Radiological Science, College of Health Science, Gachon University, Incheon, Republic of Korea. ${ }^{5}$ Department of Psychiatry, Gil Medical Center, Gachon University College of Medicine, Incheon, Republic of Korea. ${ }^{6}$ These authors contributed equally: Sang-Heon Lim and Jihyun Yoon. ${ }^{\square}$ email: kimkg@gachon.ac.kr; kangsg@gachon.ac.kr
} 
with an intra-class correlation ${ }^{11}$. Yet, this method is still time-consuming for the tracers. Overall, accurate $\mathrm{Hb}$ segmentation for quantitative analysis is still a challenging task. An accurate and quick Hb segmentation method might be a fundamental step in medical treatment, such as deep brain stimulation and neurosurgery, for targeting $\mathrm{Hb}$ sub-regions related to psychiatric diseases in the future ${ }^{12,13}$. For this reason, a couple of semi- or fully-automatic $\mathrm{Hb}$ segmentation approaches have been reported: (1) reproducibility of a myelin content-based $\mathrm{Hb}$ segmentation from $3 \mathrm{~T}$ magnetic resonance imaging (MRI) using a semi-automatic myelin contrast-based $\operatorname{method}^{14}$, and (2) a machine learning algorithm for fully-automatic Hb segmentation of 1.5 T MRI for Hb volume comparison of patients with bipolar disorder and schizophrenia with healthy controls ${ }^{15}$. Since those studies performed image processing such as intensity-based threshold and image registration ${ }^{14,15}$, there remain limitations in their ability to reliably perform automatic $\mathrm{Hb}$ segmentation in large MRI datasets. Accordingly, the development of accurate methods for an automated $\mathrm{Hb}$ segmentation of $7 \mathrm{~T}$ MRI in patients with depressive disorder is necessary. However, research on automatic analytic methods using a deep learning approach in the depressive disorder research field is currently scarce.

Recently, demonstrated as a powerful tool for semantic segmentation, deep learning methods based on convolutional neural networks can accommodate large annotated datasets and computational resources compared with traditional segmentation techniques ${ }^{16,17}$. Moreover, various studies have reported regional segmentation of the human brain and their performance using u-net-based semantic segmentation networks ${ }^{18,19}$. Nevertheless, there are no such reported cases of deep learning approaches for automated Hb segmentation. Thus, we developed a deep learning-based method for automated $\mathrm{Hb}$ segmentation using high-resolution $7 \mathrm{~T}$ MRI and assessed the clinical utility of this method using brain images of patients with MDD and NCs for the validation of our deep learning approach.

Although $7 \mathrm{~T}$ MRI is an imaging technique suitable for visualizing the $\mathrm{Hb}$, it is still challenging to segment the $\mathrm{Hb}$ accurately using naked eye-based manual segmentation because of its low anatomical contrast and tiny size, resulting in low reliability of segmentation results from different observers. To address this limitation, we designed deep learning networks trained on manual segmentation masks from two different examiners. The final $\mathrm{Hb}$ segmentation results fused the two pre-trained networks' outputs, taking into account both examiners' manually segmented masks.

Additionally, to perform automatic anatomical structure segmentation, it is more efficient to focus on specific areas of the visual scene, picking out only important features of interest, similar to human visual attention, than to examine every part of the brain. The attention u-net was designed for this purpose and has been proposed to simply and accurately segment the pancreas, which occupies a small area in the abdomen ${ }^{20,21}$. In this study, therefore, we designed our deep learning networks' architecture based on the attention u-net for robust and accurate $\mathrm{Hb}$ segmentation.

This study aimed to validate the reproducibility of our deep learning-based computer-aided tool via evaluating the automatic $\mathrm{Hb}$ segmentation performance and comparing manual and automated $\mathrm{Hb}$ volume estimation in individuals with MDD and NCs.

\section{Methods}

Study population. Patients with MDD and NCs ranging from 20 to 65 years of age were recruited from the psychiatric department of Gil Medical Center, Incheon, South Korea. At the screening evaluation, boardcertified psychiatrists had structured interviews with the participants to assess their eligibility using the standard diagnostic instrument based on the Diagnostic and Statistical Manual of Mental Disorders, 5th edition (DSM$5)^{22}$. The severity of psychiatric symptoms of all participants was measured using the Hamilton Depression Rating Scale 17 items (HDRS-17) ${ }^{23}$, Beck Depression Inventory (BDI) ${ }^{24}$, and Clinical Global Impression Scale $(\mathrm{CGI})^{25,26}$. This study was conducted in accordance with the Declaration of Helsinki and approved by the Institutional Review Board of the Gil Medical Center (IRB No. GDIRB2018-005), and written informed consent was obtained from all the participants.

The common eligibility criteria for the MDD and NC groups were as follows: (1) no previous abnormal findings on brain imaging; (2) no intellectual disability, neurocognitive disorders, or history of significant brain injury; (3) no personality disorder or substance use disorder including alcohol use disorder in the last year; (4) no major or unstable medical or neurological disorders in the last year; (5) no current serious suicide risk; (6) right-handedness using the Edinburgh Handedness Test; (7) not pregnant or lactating; and (8) no metal material in the body. The NCs were included according to the following additional criteria: (1) no family history of firstdegree relatives with a major psychiatric disorder; (2) no history or symptoms of psychiatric disorders; (3) no history of taking psychotropics during their lifetime; and (4) a total score $\leq 6$ on the HDRS-17. The participants who met the DSM-5 diagnostic criteria for $\mathrm{MDD}^{27}$ were included in the MDD group. The MDD and NC groups were matched for age and sex.

Image acquisition. Whole-brain sagittal images were acquired using an 8-channel phased-array coil for 7-T MRI (MAGNETOM 7 T, Siemens, Erlangen, Germany). To evaluate the possibility of simultaneously recording relaxation times, such as $\mathrm{T} 1$ and $\mathrm{T} 2{ }^{*}$, the prototype multi-echo magnetization-prepared 2 rapid gradient echoes (ME-MP2RAGE) sequence by Siemens was utilized ${ }^{28}$. Image acquisition was performed using the following parameters: field of view $(F O V)=166 \times 166 \times 135.2 \mathrm{~mm}^{3}$ with a nominal isotropic resolution of $0.65 \mathrm{~mm}$; matrix size $=256 \times 256 ; 208$ slices along the right-left axis (sagittal orientation); repetition time $(\mathrm{TR})=8000 \mathrm{~ms}$; two inversion times $(\mathrm{TIs})=1000 / 3200 \mathrm{~ms}$; flip angle $(\mathrm{FA})=4^{\circ}$; four echo times $(\mathrm{TEs})=3.46,7.28,11.1$, and $14.92 \mathrm{~ms}$; bandwidth $=280 \mathrm{~Hz} /$ px yielding an acquisition time $(\mathrm{TA})=14 \mathrm{~min} 16 \mathrm{~s}$; bipolar readout; generalized auto-calibrating partially parallel acquisitions with acceleration factor $=3$; and $7 / 8$ and $6 / 8$ partial Fourier factors along the phase-encoding and slice-encoding directions. 


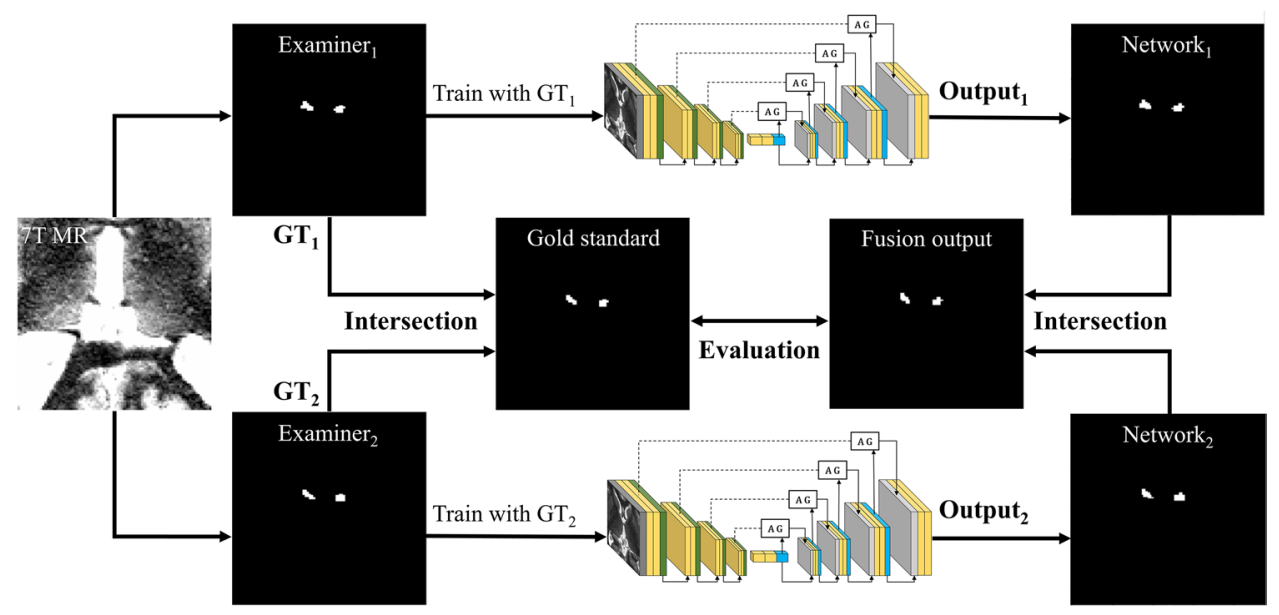

Figure 1. An illustrated overview of the automatic habenula segmentation. In the network training procedure, two manual segmentation masks were used for the training of two networks, and two segmentation results were obtained. The network evaluation was performed by comparing the intersected GT and fusion output. GT ground truth; $M R$ magnetic resonance; $7 T 7$ Tesla; $A G$ attention gate.

Label acquisition. The manual segmentation was performed by two well-trained researchers using the T1 map of the participants' 7 T MRIs. The researchers manually segmented the target voxels by tracing the Hb, which differed in signal intensity from that of the contiguous brain tissues, using three-dimensional analytic programs (i.e., Image); ver. 1.52a). We used both medial and lateral parts for manual Hb delineation (generating gold-standard).

Experimental overview. Two deep learning networks were trained for automatic Hb segmentation from the manual segmentation results of two different observers.

Figure 1 shows an overview of the network training and evaluation procedure for the automated Hb segmentation. $G T_{1}$ and $G T_{2}$ were obtained from two examiners using manual $\mathrm{Hb}$ segmentation. $G T_{1}$ was used to learn as the ground truth (GT) for Network ${ }_{1}$ and $G T_{2}$ for Network. The final automatic segmented region of the Hb was the intersection of the automatic segmentation results from two different unfamiliar networks in the test dataset. To evaluate the automatic segmentation results, the fusion of different network outputs was compared with the gold standard as presented in the Label acquisition subsection.

Preprocessing and experimental setup. We acquired a region of interest in the axial plane of the $7 \mathrm{~T}$ MRI volume (Fig. 2a). The window level and window width were set to clearly observe the Hb on 7 T MRI (window level: 1300, window width: 750) (Fig. 2b). To remove unnecessary brain regions, the images were uniformly cropped to 96 pixels (x-axis) and 128 pixels (y-axis), including the Hb (Fig. 2c,d). In this study, we excluded slices without the $\mathrm{Hb}$ to avoid a class imbalance issue. To train the segmentation network, we approximately divided the total brain MRI data $(n=69,626$ axial plane slices) by a ratio of $6: 2: 2$ (train: validation: test). The whole dataset consisted of NC and MDD participants that were balanced across all folds (Fig. 2e). The range of the number of axial slices that included the $\mathrm{Hb}$ region for individual participants was 7-11 (min-max) slices.

Network. As shown in Fig. 3, we employed an attention u-net for $\mathrm{Hb}$ segmentation, which was obtained from the source codes (https://github.com/lixiaolei1982/Keras-Implementation-of-U-Net-R2U-Net-AttentionU-Net-Attention-R2U-Net.-). The network has an encoder path followed by a decoder path, each with four resolution steps (4 depths). Each decoder or encoder path contains two convolutional blocks (2 widths). The number of filters for feature aggregation increases to $64,128,256$, or 512 depending on the depth of the network.

When the 7 T MRI was fed into the model, the significant feature maps were aggregated for Hb segmentation by the convolution operation. In the feature aggregation process, the feature map, which was reduced in resolution by a repeated pooling operation, was restored to the input image resolution by the up-sampling operation. Up-sampling was performed after the AG operation. The feed-forward procedure of the AG-based up-sampling was as follows:

$$
\begin{gathered}
A G_{\text {in }}=\sigma_{1}\left(W_{\alpha}(x)+W_{\alpha}(g)\right) \\
A G_{\text {out }}=\sigma_{2}\left(W_{s}\left(A G_{\text {in }}\right)\right) \times W_{\alpha}(g) \\
\text { Concatenate }=\operatorname{merge}\left(A G_{\text {out }}, x\right)
\end{gathered}
$$



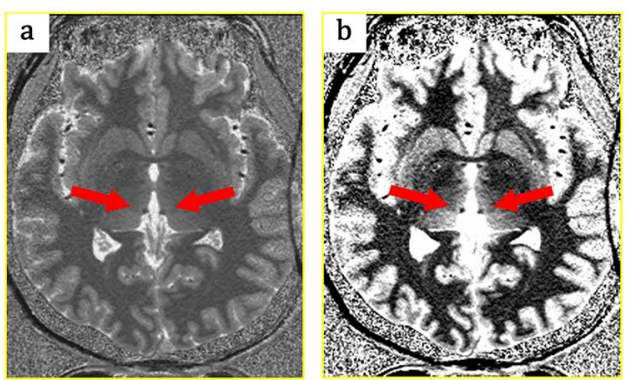

$\mathrm{e}$
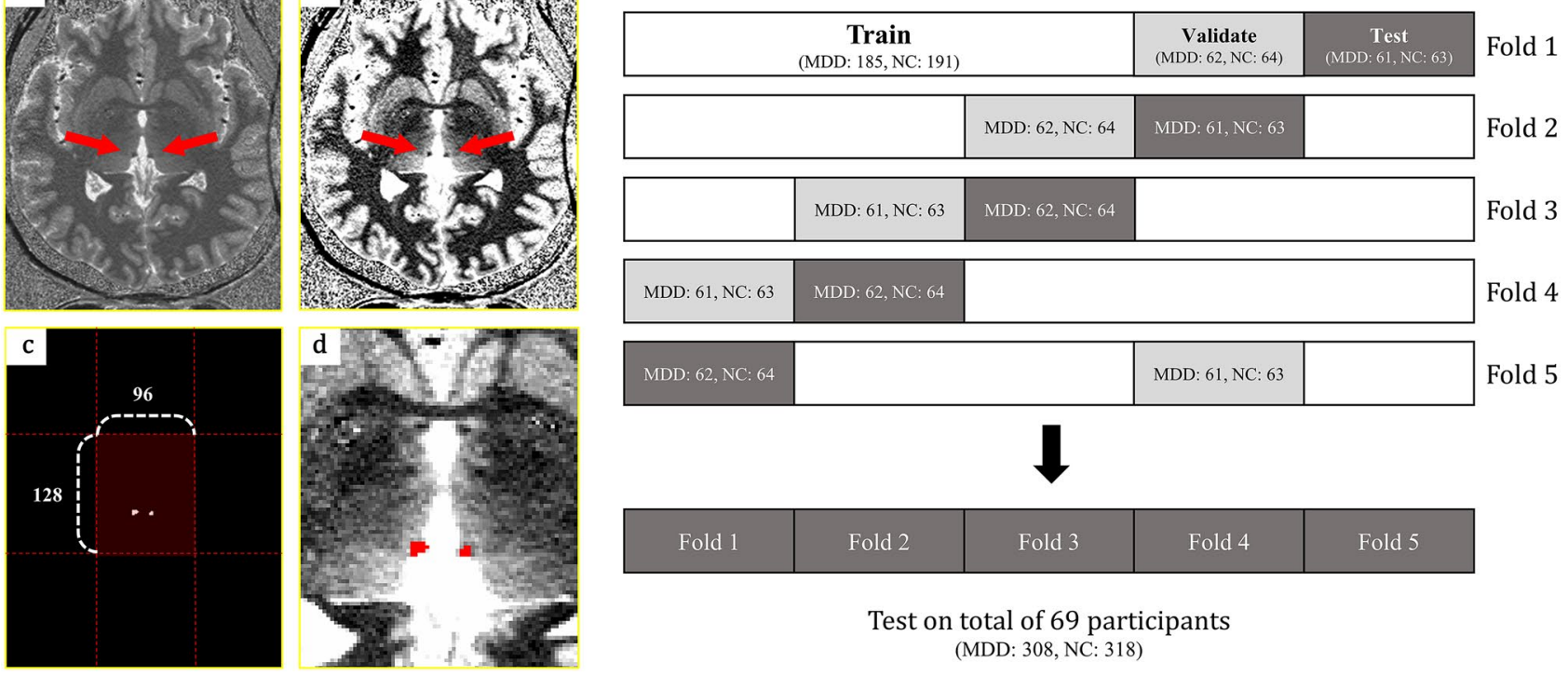

Test on total of 69 participants

(MDD: 308, NC: 318)

Figure 2. Preprocessing procedure of $7 \mathrm{~T}$ MRI (a-d), and experimental setup (e). (a) Original axial view $7 \mathrm{~T}$ MRI. (b) Window level (WL) and window width (WW) were set (WL/WW: 1300/750) to observe the Hb. (c-d) The region that included the $\mathrm{Hb}$ was coarsely chopped to the specific size. (e) An illustration of the data splitting method to conduct fivefold cross validation. MRI magnetic resonance image; $7 T 7$ Tesla; $H b$ habenula; $M D D$ major depressive disorder; NC normal control.
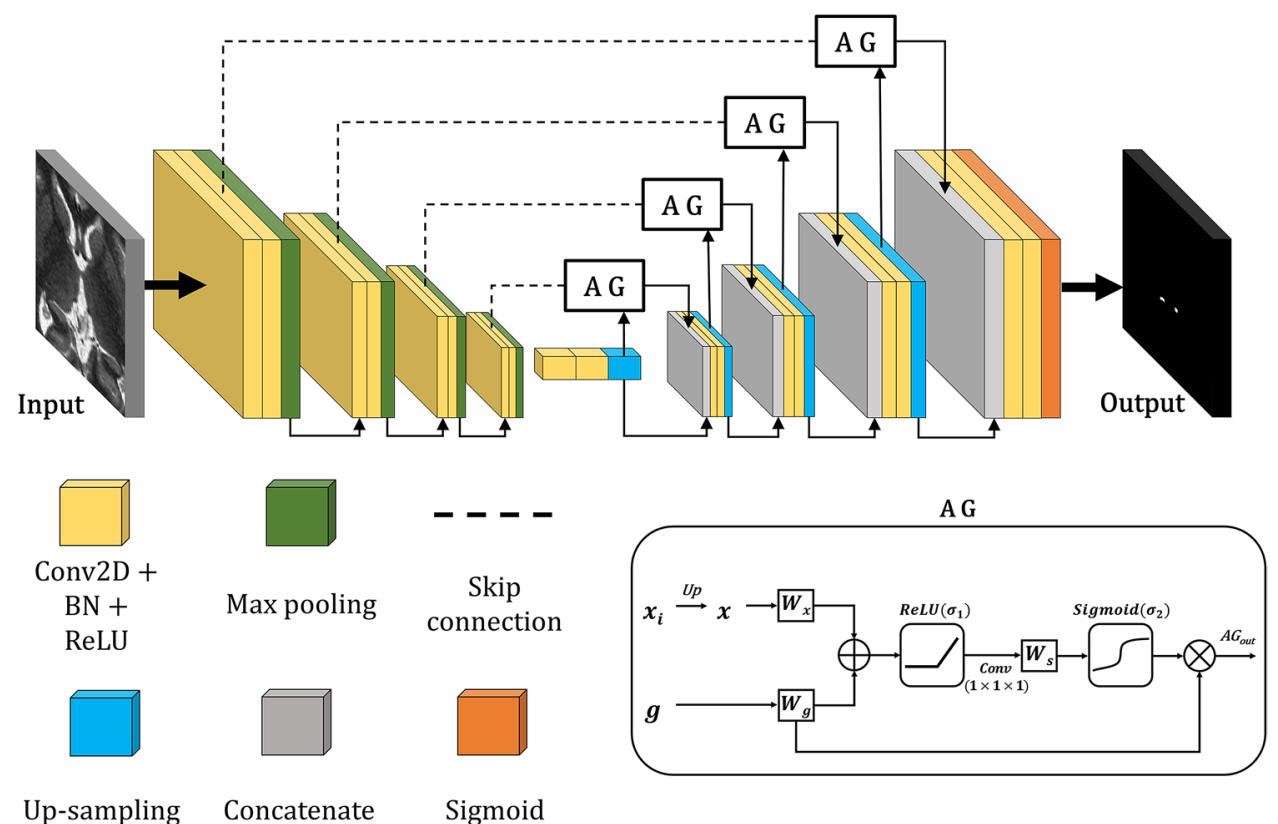

Figure 3. The architecture of the attention gate-based $\mathrm{u}$-net for $\mathrm{Hb}$ segmentation. Each colored block including the AG process is indicated below the illustration of the network architecture. The significant feature maps were aggregated by the listed convolution operations, including AG, to segment the $\mathrm{Hb}$ regions. Conv convolution; $B N$ batch normalization; $A G$ attention gate; ReLU rectified linear unit; $x$ : the feature maps of the previous layer; $g$ : the skip connected feature maps; $W$ : aggregation procedure of more than one feature with convolutional filters; $W_{s}$ : aggregation procedure of only one feature with a single convolutional filter.

where $\sigma_{1}$ is the rectified linear unit (ReLU) activation function, $\sigma_{2}$ is the sigmoid activation function, $W_{\alpha}(f)$ is a convolutional operation that maintains the number of feature map channels of $f$, and $W_{s}(f)$ is a convolutional operation that aggregates a single-channel from the $f$. The two input features maps, up-sampled feature map $x$ and skip-connected feature map $(g)$, are added, after a convolution operation $W_{\alpha}$. After summation, the added feature map $W_{\alpha}(x)+W_{\alpha}(g)$ is activated by ReLU. The single feature map aggregated by $W_{s}$ is output as an 
activation map via sigmoid activation. The output of $A G$ is a self-weighted feature map created by multiplying the activation map $\sigma_{2}\left(W_{s}\left(A G_{i n}\right)\right)$ and the skip-connected feature map $W_{\alpha}(g)$. Finally, the concatenate layer is a self-weighted feature map that focuses on the important features for segmentation of the Hb. The concatenate layer, including AG, affects the final network's output by continuing to participate in the subsequent up-sampling operation.

Implementation details. We trained our networks on a single Tesla V100 (32 GB) GPU (graphics processing unit). Each network consisted of 1,984,565 parameters. Our networks were trained using the Adam optimizer ${ }^{29}$ to jointly minimize the generalized dice $\operatorname{loss}^{30}$. We conducted early termination of the training procedure when loss did not improve during 50 epochs. The initial learning rate (LR) was 0.001 , and when the loss did not minimize for 10 epochs, the LR was reduced by a factor of 0.2 . The networks early terminated the training procedure in 100-300 epochs. In this study, we employed the following frameworks on Python (ver. 3.6.12): (1) Keras (ver. 2.2.5), TensorFlow-GPU (ver. 1.15.4) for deep learning analysis; and (2) Simple-ITK (ver. 1.2.0) for MRI preprocessing.

Statistics. Demographic data and clinical characteristics were calculated and compared using two-tailed independent t-tests and chi-square tests. The software IBM SPSS Statistics (ver. 21.0) was used and $P<0.05$ was set as the limit for statistical significance for these analyses.

We obtained the precision, recall, and dice similarity coefficient (DSC) by comparing the GT and automatic segmentation result of networks for evaluation in the test set.

To evaluate our network, we calculated the coincidence-rate of the GTs and auto-segmentation results. The evaluation was conducted voxel-wisely using the following equations:

$$
\begin{gathered}
\text { Precision }=\frac{T P}{T P+F P} \\
\text { Recall }=\frac{T P}{T P+F N} \\
D S C=\frac{2 \times \text { Precision } \times \text { Recall }}{\text { Precision }+ \text { Recall }}
\end{gathered}
$$

The true positive (TP), false positive (FP), true negative (TN), and false negative (FN) were obtained by comparing the voxels of a GT and fusion segmentation result. Since the whole dataset was divided into fivefold as a test dataset, we were able to evaluate every slice in our whole dataset (69 participants). Moreover, since deep neural networks (DNNs) are dependent on the training and validation set, we designed the training, validation, and test dataset to fivefold for every test dataset in each fold.

For the validation of clinical applications such as volume analysis, we compared manual and automated $\mathrm{Hb}$ segmentation in participants with MDD and NCs. Therefore, it was necessary to estimate the size of the Hb volume via 3D volume reconstruction for each participant (see Supplementary Fig. S1). In addition, we divided the total volume of the $\mathrm{Hb}$ into the left and right hemispheres to analyze automatic segmentation performance on each side.

After 3D reconstruction of the $\mathrm{Hb}$, the intra-class correlation coefficients (ICCs) were calculated from each pair of brain volumes using the automatic and manual segmentation methods. Before this analysis, the normalization of the $\mathrm{Hb}$ volumes was performed using total intracranial volume (ICV). The Hb volumes were divided by the ICV for each participant $\left(\frac{H b \text { Volume }}{I C V} \times 100 \%\right)$ to adjust for individual differences in brain size. After obtaining the number of voxels in the mask, the ICV was calculated by multiplying by the resolution. To make one mask, each subject's gray matter, white matter, and CSF from the 3 T MRI were segmented using SPM12 (see http://www.fil.ion.ucl.ac.uk/spm) and then summed. To assess the inter-rater reliability (i.e., the degree of agreement between the $\mathrm{Hb}$ volumes by automatic and manual segmentation), the ICC method involving the absolute agreement mode, which is sensitive to the differences in the mean values of observations, was used ${ }^{31,32}$. The reliability ICC $\left(r_{I C C}\right)$ values were interpreted according to Cicchetti's guidelines ${ }^{33}$ as follows: $<0.40$, poor; $0.40-0.59$, fair; 0.60-0.74, good; and 0.75-1.00, excellent. SPSS was used for the ICC analysis.

\section{Results}

Demographics. Supplementary Table S1 shows the demographics of the participants in this study. The age and sex ratio did not significantly differ between the two groups. The years of education and depressive symptom severity measured using the HDRS-17, BDI, and CGI differed significantly between the two groups.

Evaluation of habenula segmentation. The average total number of voxels with automated segmentation for the $\mathrm{Hb}$ out of all voxels $(256 \times 256 \times 208)$ was $24.01 \pm 6.42 \mathrm{~mm}^{3}$ (mean \pm standard deviation), and in the case of manual segmentation it was $24.19 \pm 6.10 \mathrm{~mm}^{3}$. We divided the Hb volume into the left and right hemispheres in the MDD and NC groups. The Hb volumes from manual and automated segmentation in both groups were as follows: (1) left $\mathrm{Hb}$ in $\mathrm{NC}, 12.40 \pm 4.00 \mathrm{~mm}^{3}$ and $11.98 \pm 4.06 \mathrm{~mm}^{3}$ (manual method and automated method); (2) right $\mathrm{Hb}$ in NC, $12.21 \pm 3.57 \mathrm{~mm}^{3}$ and $11.79 \pm 3.26 \mathrm{~mm}^{3}$; (3) left $\mathrm{Hb}$ in MDD, $12.52 \pm 3.08 \mathrm{~mm}^{3}$ and $12.58 \pm 3.34 \mathrm{~mm}^{3}$; (4) right $\mathrm{Hb}$ in MDD, $11.21 \pm 3.16 \mathrm{~mm}^{3}$ and $11.69 \pm 2.82 \mathrm{~mm}^{3}$. The volumes from automated segmentation tended to be underestimated in NC participants and overestimated in MDD participants when 


\begin{tabular}{|l|l|l|l|}
\hline & Precision & Recall & DSC \\
\hline NC participants $(\mathbf{n}=\mathbf{3 6})$ \\
\hline Left $\mathrm{Hb}$ & $0.885 \pm 0.112$ & $0.853 \pm 0.176$ & $0.847 \pm 0.145$ \\
\hline Right $\mathrm{Hb}$ & $0.889 \pm 0.156$ & $0.871 \pm 0.152$ & $0.862 \pm 0.127$ \\
\hline Total $\mathrm{Hb}$ & $0.883 \pm 0.120$ & $0.862 \pm 0.109$ & $0.863 \pm 0.079$ \\
\hline MDD participants $(\mathbf{n}=\mathbf{3 3})$ \\
\hline Left $\mathrm{Hb}$ & $0.868 \pm 0.190$ & $0.872 \pm 0.191$ & $0.855 \pm 0.162$ \\
\hline Right $\mathrm{Hb}$ & $0.819 \pm 0.198$ & $0.867 \pm 0.188$ & $0.813 \pm 0.158$ \\
\hline Total $\mathrm{Hb}$ & $0.848 \pm 0.127$ & $0.866 \pm 0.149$ & $0.842 \pm 0.120$ \\
\hline Total participants $(\mathbf{n}=\mathbf{6 9})$ \\
\hline Left $\mathrm{Hb}$ & $0.877 \pm 0.175$ & $0.862 \pm 0.170$ & $0.860 \pm 0.147$ \\
\hline Right $\mathrm{Hb}$ & $0.854 \pm 0.176$ & $0.868 \pm 0.173$ & $0.846 \pm 0.136$ \\
\hline Total $\mathrm{Hb}$ & $0.869 \pm 0.124$ & $0.865 \pm 0.134$ & $0.852 \pm 0.094$ \\
\hline
\end{tabular}

Table 1. The performance evaluation of the automatic segmentation results using the intersection network. The evaluation results are presented as mean and standard deviation. $\mathrm{Hb}$ habenula, DSC dice similarity coefficient.

compared to the manually segmented volumes. In the Pearson's correlations of the clinical variables with the $\mathrm{Hb}$ volumes of the MDD group, there were no significant correlations: (1) HDRS-17: $r=0.158, p=0.381$; (2) BDI: $r=0.065, p=0.718$; (3) CGI: $r=-0.037, p=0.838$.

In addition, the average $\mathrm{T} 1$ value was $105,050 \pm 27,476$ (mean \pm standard deviation) for automated segmentation and $106,395 \pm 26,433$ for manual segmentation, which was not significantly different between the two groups (paired $t$-test: NC group, $p=0.145$; MDD group, $p=0.578$ ).

Table 1 shows the performance evaluation of the automated $\mathrm{Hb}$ segmentation. The performance of our network reached a mean precision, recall, and DSC of $0.869,0.865$, and 0.852 , respectively, using fivefold cross validation. The evaluation results of individual networks for fusion segmentation output are presented in Supplementary Table S2. The performance of Network $k_{1}$ using $G T_{1}$ as the reference image reached a mean precision, recall, and DSC of $0.848,0.817$, and 0.815 , respectively. Furthermore, the performance of Network $k_{2}$ reached a mean precision, recall, and DSC of $0.852,0.825$, and 0.818 , respectively. We also trained a single attention u-net from the intersected GT of the two raters for comparison with the proposed network. It achieved a mean precision, recall, and DSC of 0.847, 0.789, and 0.790, respectively. The proposed network achieved a higher recall than did the network that did not consider the two raters' manual segmentation results (see Supplementary Table S3 \& Fig. S2).

Figure 4 shows a cross-sectional view of the segmentation results of several cases. The segmentation performance of the automated segmentation model was highly consistent with GT. The cross validation results showed that the segmentation performance was similar for all folds, and there was a low standard deviation in each fold.

In addition, we conducted twofold cross validation to investigate the bias from using both MDD and NC data together for dataset generation. We separated total images into two folds: MDD participants for the training dataset and NC participants for the test dataset, and vice versa. Mann-Whitney U-tests showed significant differences in the mean precision $(p<0.001)$ and recall $(p<0.001)$ between the individual folds (Supplementary Table S4). In contrast, there was no significant difference $(p=0.283)$ between the mean DSCs from either fold.

Intra-class correlation analysis. Table 2 shows the results of intra-class correlation analyses between automated and manual segmentation of the $\mathrm{Hb}$ volume. The mean of the normalized total $\mathrm{Hb}$ volume with automatic segmentation was $16.66 \times 10^{-4} \pm 4.39 \times 10^{-4} \%$, and the mean of the normalized total Hb volume with manual segmentation was $16.77 \times 10^{-4} \pm 4.05 \times 10^{-4} \%$. The ICCs calculating the agreement between the Hb volumes by automatic and manual segmentation were excellent for the MDD group $\left(r_{I C C}=0.818, p<0.001, \mathrm{n}=33\right)$, NC group $\left(r_{I C C}=0.897, p<0.001, \mathrm{n}=36\right)$, and participants overall $\left(r_{I C C}=0.870, p<0.001, \mathrm{n}=69\right)$.

Significant ICCs were obtained for both the left and right $\mathrm{Hb}$. In the left Hb, excellent ICCs were observed in the MDD group $\left(r_{I C C}=0.920, p<0.001\right)$, NC group $\left(r_{I C C}=0.903, p<0.001\right)$, and participants overall $\left(r_{I C C}=0.908\right.$, $p<0.001)$. In the right $\mathrm{Hb}$, we obtained excellent ICC values in the NC group $\left(r_{I C C}=0.819, p<0.001\right)$ and participants overall $\left(r_{I C C}=0.750, p<0.001\right)$, and a good ICC value for the MDD group $\left(r_{I C C}=0.658, p=0.001\right)$. The Bland-Altman analyses of the estimated $\mathrm{Hb}$ volumes from automatic and manual segmentation in the MDD and NC groups are presented in Fig. 5. The Bland-Altman plots show the reproducibility of the automatic $\mathrm{Hb}$ segmentation method for $\mathrm{Hb}$ volume estimation.

\section{Discussion}

In this study, we proposed a deep attention u-net-based intersection network for accurate Hb segmentation and quantitative $\mathrm{Hb}$ analysis. As a result of experiments, the mean precision, recall, and DSC in the automatic segmentation using the intersection of attention u-net was good in the total participants. Additionally, the ICCs between automatic and manual segmentation of the total $\mathrm{Hb}$ were excellent in all participants, participants with $\mathrm{MDD}$, and NCs. Therefore, we suggest that the proposed approach is suitable for the segmentation of the $\mathrm{Hb}$, which is a brain region tiny in size with low contrast in brain MRI. To the best of our knowledge, this is the first 

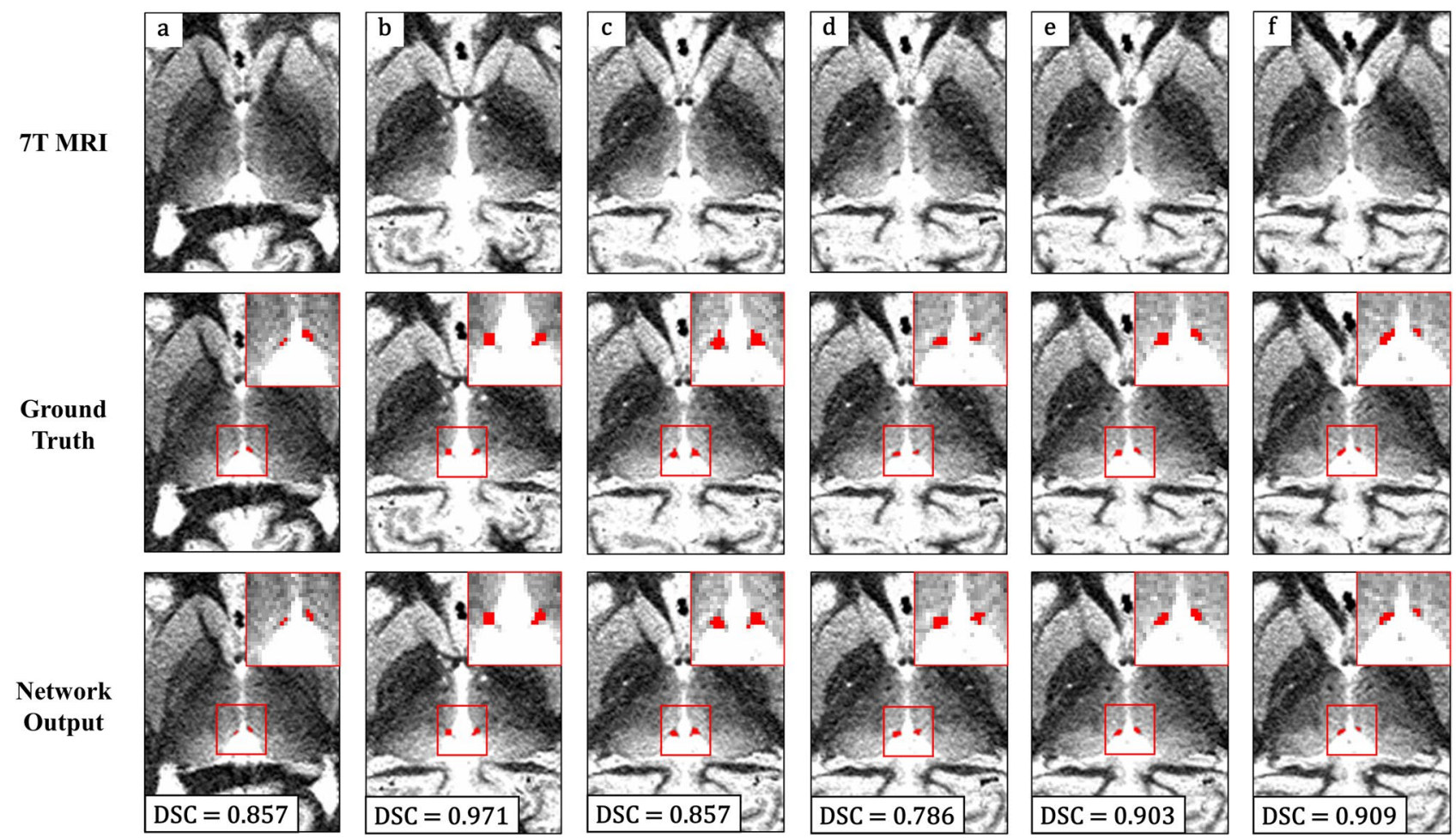

Figure 4. Segmentation results of several test cases (a-f). Presented MRIs were set to window level, 1300 and window width, 750 (top row). The ground truth is the intersection of two examiners (middle row). The prediction result is the intersection of the two trained segmentation networks (bottom row). Both ground truth and the automated segmentation results are presented as overlays on cross-sectional 7 T MRIs. DSC dice similarity coefficient; MRI magnetic resonance imaging; 7 T 7 Tesla.

\begin{tabular}{|c|c|c|c|c|}
\hline & \multicolumn{2}{|l|}{ Habenula volume $^{\mathrm{a}}$} & \multicolumn{2}{|c|}{ Intra-class correlation } \\
\hline & Automatic estimation & Manual estimation & ICC & $p$-value \\
\hline \multicolumn{5}{|c|}{ NC $(n=36)$} \\
\hline Left $\mathrm{Hb}$ & $8.11 \times 10^{-4} \pm 2.91 \times 10^{-4}$ & $8.55 \times 10^{-4} \pm 2.79 \times 10^{-4}$ & 0.903 & $<0.001$ \\
\hline Right $\mathrm{Hb}$ & $7.89 \times 10^{-4} \pm 2.37 \times 10^{-4}$ & $8.30 \times 10^{-4} \pm 2.31 \times 10^{-4}$ & 0.819 & $<0.001$ \\
\hline Total $\mathrm{Hb}$ & $16.00 \times 10^{-4} \pm 5.04 \times 10^{-4}$ & $16.85 \times 10^{-4} \pm 4.52 \times 10^{-4}$ & 0.897 & $<0.001$ \\
\hline \multicolumn{5}{|c|}{$\operatorname{MDD}(n=33)$} \\
\hline Left $\mathrm{Hb}$ & $8.93 \times 10^{-4} \pm 2.05 \times 10^{-4}$ & $8.72 \times 10^{-4} \pm 1.99 \times 10^{-4}$ & 0.920 & $<0.001$ \\
\hline Right $\mathrm{Hb}$ & $8.45 \times 10^{-4} \pm 1.88 \times 10^{-4}$ & $7.96 \times 10^{-4} \pm 2.31 \times 10^{-4}$ & 0.658 & 0.001 \\
\hline Total Hb & $17.38 \times 10^{-4} \pm 3.49 \times 10^{-4}$ & $16.68 \times 10^{-4} \pm 3.54 \times 10^{-4}$ & 0.818 & $<0.001$ \\
\hline \multicolumn{5}{|c|}{ Total participants $(n=69)$} \\
\hline Left $\mathrm{Hb}$ & $8.50 \times 10^{-4} \pm 2.55 \times 10^{-4}$ & $8.63 \times 10^{-4} \pm 2.42 \times 10^{-4}$ & 0.908 & $<0.001$ \\
\hline Right $\mathrm{Hb}$ & $8.16 \times 10^{-4} \pm 2.15 \times 10^{-4}$ & $8.14 \times 10^{-4} \pm 2.30 \times 10^{-4}$ & 0.750 & $<0.001$ \\
\hline Total $\mathrm{Hb}$ & $16.66 \times 10^{-4} \pm 4.39 \times 10^{-4}$ & $16.77 \times 10^{-4} \pm 4.05 \times 10^{-4}$ & 0.870 & $<0.001$ \\
\hline
\end{tabular}

Table 2. The intra-class correlation analysis between automatic and manually segmented habenula volumes. Significant results are indicated in bold. SD standard deviation, ICC intra-class correlation coefficient. ${ }^{a}$ Habenula volumes were normalized using total intracranial volume (ICV). Habenula volumes were divided by the ICV for each participant as a normalization process (regional brain volume/ ICV $\times 100 \%$ ) for the subsequent analysis. Normalized habenula volumes are described as mean $\pm \mathrm{SD}$.

study which presented an automatic Hb segmentation based on volume estimation method in participants with MDD and NCs using 7 T MRI.

In the automatic segmentation procedure, we obtained a mean precision, recall, and DSC of $0.869,0.865$, and 0.852 , respectively. In recent years, a couple of studies on automated segmentation of the Hb have been reported. The first study performed semi- and fully-automated segmentation in $3 \mathrm{~T}$ MRI of healthy young adults $^{14}$, and the second study performed fully-automated segmentation in children, adolescents, and adults 

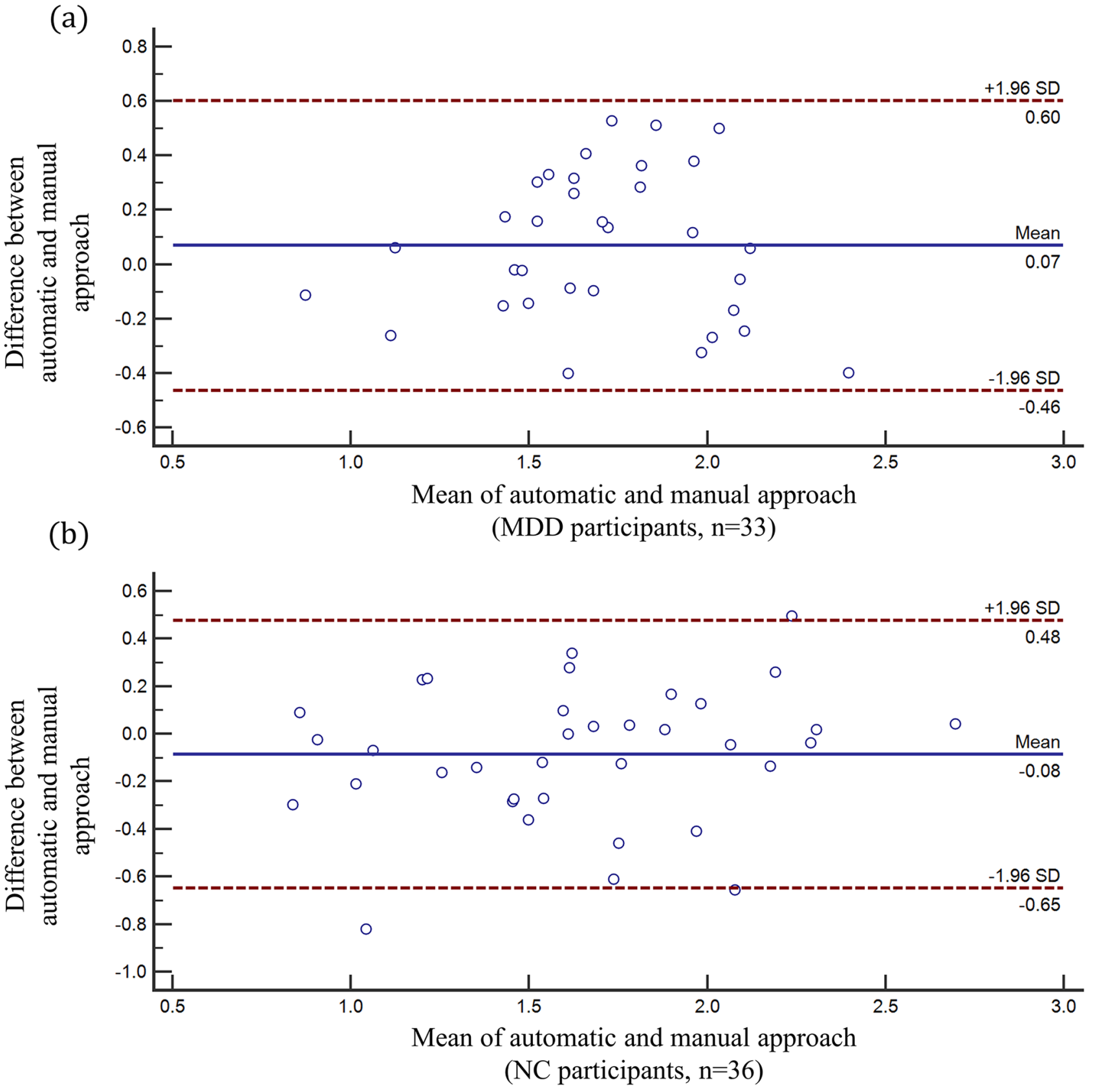

Figure 5. Bland-Altman analysis assessing the reproducibility of the automatic habenula segmentation method for (a) MDD participants and (b) NC participants. Habenula volumes were divided by the intracranial volume (ICV) for each participant (regional brain volume/ ICV $\times 100 \%$ ) as a normalization process for the subsequent analysis. $M D D$ major depressive disorder; $N C$ normal control; SD standard deviation.

with bipolar disorder and schizophrenia ${ }^{15}$. In the first study, the DSC for binary segmentation reached 0.71 for semi-automated segmentation and 0.69 for fully-automated segmentation, and the DSC of the probability map reached 0.74 for both semi- and fully-automated segmentation ${ }^{14}$. In a more recent study that segmented the $\mathrm{Hb}$ with a fully-automated framework, the DSC of the inter-rater reliability tests between manual and automatic segmentation ranged between 0.758 and $0.828^{15}$. Although the participants in the previous studies had different clinical characteristics from those in our study, our automatic $\mathrm{Hb}$ segmentation approach seems to be more accurate (mean DSC $>0.85$ ) than that of the other studies.

In this study, the $r_{I C C}$ of the total $\mathrm{Hb}$ ranged between 0.818 and 0.897 , depending on the group. In a previous study conducted on healthy young adults, the $r_{I C C}$ for the Hb was 0.62 for semi-automated segmentation and 0.47 for fully-automated segmentation ${ }^{14}$, which shows the superiority of our approach. However, the $r_{I C C}$ of the $\mathrm{Hb}$ was different between the groups and hemispheres in our study. According to the ICC analysis, the $r_{I C C}$ of the left $\mathrm{Hb}$ was excellent (0.903-0.920), while the $r_{I C C}$ of the right Hb was from 0.658 (MDD) to 0.819 (NC). Specifically, the volume estimation performance for the right Hb of MDD participants was slightly lower than that of NC participants. With respect to the right $\mathrm{Hb}$, the volume in some of the MDD participants was low, 
which might be one reason for the discrepancy between the NC and MDD groups, both in the Hb segmentation and in the volume estimation. In addition, the asymmetry of the left and right $\mathrm{Hb}$ also might be a reason ${ }^{34}$.

Another attribute of our approach is that the results of two networks, each trained on GT generated by two different observers, were intersected to output fusion segmentation results. When trained with the intersected GT, the network reached a low mean recall (0.789) compared to that of the single attention-network (0.865). Since the $\mathrm{Hb}$ is a very small region in the brain, there may be slight differences in the manual delineation results from two independent raters. Accordingly, we assume that training and intersecting independent networks according to individual raters (i.e., $G T_{1}$ and $G T_{2}$ ) would achieve more reliable segmentation results than a single network that encoded both raters (e.g., union or intersection of GTs).

Our approach is different from the previous studies for following reasons: first, this is the first automated segmentation study performed in participants with MDD and NCs using high-resolution 7 T MRI that can ideally visualize the $\mathrm{Hb}$. Second, a DNNs approach for automatic $\mathrm{Hb}$ segmentation and volume estimation was conducted. We designed a deep learning network based on the attention u-net that was optimized for segmenting small objects (i.e., the $\mathrm{Hb}$ ) of various shapes. Third, since the segmentation was performed by the fusion of the two pre-trained attention u-net using two different GTs, it is believed that a more reliable segmentation was achieved.

We investigated whether there was bias in our network training procedure that included both MDD and NC participants. There were two limitations in this bias check process: (1) an imbalance in the number of images in the training and test sets, and (2) a decrease in the number of images in the training set. However, for the bias check, we divided the total images into an MDD dataset and an NC dataset (MDD, 308 axial slices; NC, 318 axial slices), assessing the results when using one dataset for training and the other for testing. We obtained significantly different results in the mean precision and recall, but not DSC, between the individual folds. However, further study is necessary to investigate potential bias using a large 7 T MRI dataset.

The high DSC and reproducibility of the automated segmentation of this study demonstrate that the applicability of the DNNs' approach for Hb volume estimation in 7 T MRI is promising. Although the Hb is considered to be an important brain region in the etiology of major psychiatric disorders, its small size has made it difficult to investigate via neuroimaging. The $\mathrm{Hb}$ is involved in emotional and cognitive processes, having connections to many other areas of the brain (e.g., thalamus, prefrontal cortex, basal ganglia, and brainstem monoaminergic neurotransmitter systems $)^{35,36}$. Recently, there are many studies focused on the connectivity between the $\mathrm{Hb}$ and other brain regions of interest such as monoamine centers and the thalamus in depression ${ }^{37,38}$. However, manual segmentation is time-consuming, highly variable, and the rater must acquire a high level of technical ability and anatomical knowledge for accurate segmentation, which has become a significant barrier to entry into this field of research ${ }^{15}$.

Considering that the data acquired through neuroimaging research is gradually increasing and that machine learning techniques are becoming more popular, the automatic segmentation approach in our study is expected to be a useful tool for many future studies.

\section{Conclusion}

This study presented an intersection network based on attention u-net for an automated segmentation of the $\mathrm{Hb}$ using 7 T MRI that performed automatic segmentation and estimated the Hb volume with high accuracy and reproducibility (i.e., high DSC and correlation coefficients). Furthermore, it is expected that the proposed automatic $\mathrm{Hb}$ segmentation method will be useful for future psychiatric neuroimaging studies to facilitate automatic segmentation and volume estimation of the $\mathrm{Hb}$ and other important small brain regions in $7 \mathrm{~T}$ MRI.

\section{Data availability}

The datasets generated during or analyzed during the current study are available from the corresponding author on reasonable request.

Received: 28 January 2021; Accepted: 18 June 2021

Published online: 29 June 2021

\section{References}

1. Hikosaka, O., Sesack, S. R., Lecourtire, L. \& Shepard, P. D. Habenula: crossroad between the basal ganglia and the limbic system. J. Neurosci. 28, 11825-11829 (2008).

2. Poller, W. C. et al. Lateral habenular neurons projecting to reward-processing monoaminergic nuclei express hyperpolarizationactivated cyclic nucleotid-gated cation channels. Neuroscience 193, 205-216 (2011).

3. Batalla, A. et al. The role of the habenula in the transition from reward to misery in substance use and mood disorders. Neurosci. Biobehav. Rev. 80, 276-285 (2017).

4. Lecca, S., Meye, F. J. \& Mameli, M. The lateral habenula in addiction and depression: An anatomical, synaptic and behavioral overview. Eur. J. Neurosci. 39, 1170-1178 (2014).

5. Ranft, K. et al. Evidence for structural abnormalities of the human habenular complex in affective disorders but not in schizophrenia. Psychol. Med. 40, 557-567 (2009).

6. Savitz, J. B., Rauch, S. L. \& Drevets, W. C. Reproduced from Habenula volume in bipolar disorder and major depressive disorder: A high-resolution magnetic resonance imaging study. Mol. Psychiatry. 18, 523 (2013).

7. Carceller-Sindreu, M. et al. Volumetric MRI study of the habenula in first episode, recurrent and chronic major depression. Eur. Neuropsychopharmacol. 25, 2015-2021 (2015).

8. Savitz, J. B. et al. Habenula volume in bipolar disorder and major depressive disorder: A high-resolution magnetic resonance imaging study. Biol. Psychiatry. 69, 336-343 (2011).

9. Schmidt, F. M. et al. Habenula volume increases with disease severity in unmedicated major depressive disorder as revealed by $7 \mathrm{~T}$ MRI. Eur. Arch. Psychiatry Clin. Neurosci. 267, 107-115 (2017).

10. Lawson, R. P., Drevets, W. C. \& Roiser, J. P. Defining the habenula in human neuroimaging studies. Neuroimage 64, 722-727 (2013). 
11. Pantel, J. et al. A new method for the in vivo volumetric measurement of the human hippocampus with high neuroanatomical accuracy. Hippocampus 10, 752-758 (2000).

12. Akram, H. et al. Subthalamic deep brain stimulation sweet spots and hyperdirect cortical connectivity in Parkinson's disease. Neuroimage 158, 332-345 (2017).

13. Morris, L. S. et al. Ultra-high field MRI reveals mood-related circuit disturbances in depression: A comparison between 3-Tesla and 7-Tesla. Transl. Psychiatry 9, 1-11 (2019).

14. Kim, J. W. et al. Reproducibility of myelin content-based human habenula segmentation at 3 Tesla. Hum. Brain Mapp. 39, 3058-3071 (2018).

15. Germann, J. et al. Fully automated habenula segmentation provides robust and reliable volume estimation across large MRI datasets suggesting intriguing developmental trajectories in psychiatric disease. Biol. Psychiatry Cogn. Neurosci. Neuroimaging. 5, 923-929 (2020).

16. Shelhamer, E., Long, J. \& Darrell, T. Fully convolutional networks for semantic segmentation. IEEE Trans. Pattern Anal. Mach. Intell. 39, 640-651 (2017).

17. LeCun, Y. \& Bengio, Y. Convolutional networks for images, speech, and time series.(ed. Arbib, M.A.). 255-258 (MIT press, 1995).

18. Nguyen, D.M.H., Vu, H.T., Ung, H.Q. \& Nguyen, G.T. 3D-brain segmentation using deep neural network and gaussian mixture model. in proceedings of the IEEE Winter Conference on Applications of Computer Vision. 815-824 (IEEE, 2017).

19. Ding, Z., Han, X. \& Niethammer, M. Votenet +: An Improved Deep Learning Label Fusion Method for Multi-Atlas Segmentation. In proceedings of 2020 IEEE International Symposium on Biomedical Imaging. 363-367 (IEEE, 2020).

20. Oktay, O., et al. Attention u-net: learning where to look for the pancreas. In proceedings of the Conference on Medical Imaging with Deep Learning. (MIDL, 2018).

21. Anderson, P., et al. Bottom-up and top-down attention for image captioning and visual question answering. In Proceedings of the IEEE conference on computer vision and pattern recognition. 6077-6086 (IEEE, 2018).

22. First, M., et al. Structured Clinical Interview for DSM-5-Research Version (SCID-5 for DSM-5, Research Version; SCID-5-RV) 1-94 (American Psychiatric Association, 2015).

23. Yi, J. S. et al. Validity and reliability of the Korean version of the Hamilton Depression Rating Scale (K-HDRS). J. Korean Neuropsychiatr. Assoc. 44, 456-465 (2005).

24. Han, H. M. et al. Korean standardization study of Beck Depression Inventory in Korea. J. Korean Neuropsychiatric Assoc. 25, 487-502 (1986).

25. Busner, J. \& Targum, S. D. The clinical global impressions scale: Applying a research tool in clinical practice. Psychiatry 4, 28 (2007).

26. Haro, J. M. et al. The Clinical Global Impression-Schizophrenia scale: A simple instrument to measure the diversity of symptoms present in schizophrenia. Acta Psychiatr. Scand. Suppl. 107, 16-23 (2003).

27. American Psychiatric Association. Diagnostic and statistical manual of mental disorders (DSM-5 ${ }^{\circledR}$ ) (American Psychiatric Association, 2013).

28. Metere, R., Kover, T., Möller, H. E. \& Schäfer, A. Simultaneous quantitative MRI mapping of T1, T2* and magnetic susceptibility with multi-echo MP2RAGE. PLoS ONE 12, e0169265. https://doi.org/10.1371/journal.pone.0169265 (2017).

29. Kingma, D.P. \& Ba, J. Adam: A method for stochastic optimization. In proceedings of the International Conference on Learning Representations. (ICLR, 2014).

30. Sudre, C.H., Li, W., Vercauteren, T., Ourselin, S. \& Cardoso, M. Generalised dice overlap as a deep learning loss function for highly unbalanced segmentation. in proceeding of the International Workshop on Deep Learning in Medical Image Analysis. 10553, 240-248 (Springer, 2017).

31. Fisher, R.A. Statistical Methods, Experimental Design, and Scientific Inference (ed. Bennett J.H.) (Oxford University Press, 1990).

32. Fisher, R.A. Statistical Methods for Research Workers. 356 (Oliver and Boyd, 1954).

33. Cicchetti, D. V. Guidelines, criteria, and rules of thumb for evaluating normed and standardized assessment instruments in psychology. Psychol. Assess. 6, 284-290 (1994).

34. Ahumada-Galleguillos, P. et al. Directional asymmetry in the volume of the human habenula. Brain Struct. Funct. 222, 1087-1092 (2017).

35. Benarroch, E. E. Habenula: Recently recognized functions and potential clinical relevance. Neurology 85, 992-1000 (2015).

36. Boulos, L. J., Darcq, E. \& Kieffer, B. L. Translating the habenula-from rodents to humans. Biol Psychiatry. 81, 296-305 (2017).

37. Ely, B. A. et al. Resting-state functional connectivity of the human habenula in healthy individuals: Associations with subclinical depression. Hum. Brain Mapp. 37, 2369-2384 (2016).

38. Luan, S. X., Zhang, L., Wang, R., Zhao, H. \& Liu, C. A resting-state study of volumetric and functional connectivity of the habenular nucleus in treatment-resistant depression patients. Brain Behav. 9, 1229. https://doi.org/10.1002/brb3.1229 (2019).

\section{Acknowledgements}

This work was supported by a National Research Foundation of Korea (NRF) Grant funded by the Korean government (MSIT) (Grant Number: NRF-2020R1A2C1007527) and by Gachon University (2019-0369).

\section{Author contributions}

S.H.L. conducted the deep learning analysis, drafted the manuscript, and performed statistical analyses. J.H.Y. drafted and revised the manuscript. Y.J.K. and C.K.K. advised the study design and the automatic and manual segmentations. S.E.C. recruited the participants and performed statistical analyses. K.G.K. designed the study, supervised the deep learning analysis, and revised the manuscript. S.G.K. designed the study, recruited the participants, performed statistical analyses, and revised the manuscript.

\section{Competing interests}

The authors declare no competing interests.

\section{Additional information}

Supplementary Information The online version contains supplementary material available at https://doi.org/ 10.1038/s41598-021-92952-z.

Correspondence and requests for materials should be addressed to K.G.K. or S.-G.K.

Reprints and permissions information is available at www.nature.com/reprints.

Publisher's note Springer Nature remains neutral with regard to jurisdictional claims in published maps and institutional affiliations. 
(c) (i) Open Access This article is licensed under a Creative Commons Attribution 4.0 International cc) License, which permits use, sharing, adaptation, distribution and reproduction in any medium or format, as long as you give appropriate credit to the original author(s) and the source, provide a link to the Creative Commons licence, and indicate if changes were made. The images or other third party material in this article are included in the article's Creative Commons licence, unless indicated otherwise in a credit line to the material. If material is not included in the article's Creative Commons licence and your intended use is not permitted by statutory regulation or exceeds the permitted use, you will need to obtain permission directly from the copyright holder. To view a copy of this licence, visit http://creativecommons.org/licenses/by/4.0/.

(C) The Author(s) 2021 